\title{
Yersinia pestis infection and laboratory conditions alter flea-associated bacterial communities
}

\author{
Ryan T Jones $^{1}$, Sara M Vetter ${ }^{2}$, John Montenieiri, Jennifer Holmes, Scott A Bernhardt ${ }^{3}$ \\ and Kenneth L Gage \\ Division of Vector-Borne Disease, Centers for Disease Control and Prevention, Fort Collins, CO, USA
}

\begin{abstract}
We collected Oropsylla montana from rock squirrels, Spermophilus varigatus, and infected a subset of collected fleas with Yersinia pestis, the etiological agent of plague. We used bar-tagged DNA pyrosequencing to characterize bacterial communities of wild, uninfected controls and infected fleas. Bacterial communities within $Y$. pestis-infected fleas were substantially more similar to one another than communities within wild or control fleas, suggesting that infection alters the bacterial community in a directed manner such that specific bacterial lineages are severely reduced in abundance or entirely eliminated from the community. Laboratory conditions also significantly altered flea-associated bacterial communities relative to wild communities, but much less so than $Y$. pestis infection. The abundance of Firmicutes decreased considerably in infected fleas, and Bacteroidetes were almost completely eliminated from both the control and infected fleas. Bartonella and Wolbachia were unaffected or responded positively to $Y$. pestis infection.

The ISME Journal (2013) 7, 224-228; doi:10.1038/ismej.2012.95; published online 16 August 2012

Subject Category: microbe-microbe and microbe-host interactions

Keywords: Bartonella; experimental infection; plague
\end{abstract}

Plague is among the most notorious diseases; it is globally widespread and epidemics are common, especially in the developing world (Gage and Kosoy, 2005). Fleas vector Yersinia pestis, the etiological agent of plague, and determining factors that affect vector competency will help develop effective strategies to control and prevent disease spread. Flea species differ in their ability to transmit $Y$. pestis, and morphological differences, particularly among proventriculi, may be partly responsible (Eskey and Haas, 1940; Korzun and Nikitin, 1997). However, the ability of $Y$. pestis to persist within a flea varies among individuals within a flea species, suggesting that morphology alone cannot explain variance in vector competency.

Flea-associated bacterial communities vary between individuals within a species (Jones et al., 2012) among discrete populations of the same species (Jones et al., 2010), and across time (Jones et al.,

Correspondence: RT Jones, Department of Environmental Sciences, Faculty of Agriculture and Environment, University of Sydney, Biomedical Building, Eveleigh, NSW 2015, Australia.

E-mail: DrRyanJones@gmail.com

${ }^{1}$ Current address: Department of Environmental Sciences, Faculty of Agriculture and Environment, University of Sydney, Biomedical Building, Eveleigh, NSW 2015, Australia.

${ }^{2}$ Current address: Virology/Immunology Unit, Public Health Laboratory Division, Minnesota State Health Department, 601 Roberts Street N, St. Paul, MN 55155, USA.

${ }^{3}$ Current address: Department of Biology, Utah State University, 5305 Old Main Hill, Logan, UT 84322, USA

Received 9 February 2012; revised 9 July 2012; accepted 10 July 2012; published online 16 August 2012
2010). These differences in flea-associated bacteria may in turn help explain why individual fleas differ in their ability to transmit Y. pestis, why some fleas are able to clear infections, why plague outbreaks are patchily distributed across the landscape and why plague occurrence is episodic. Determining how $Y$. pestis and other flea-associated bacteria interact is critical to a more thorough understanding of plague ecology, and may ultimately lead to better prediction of plague outbreaks.

The primary vector of $Y$. pestis to humans in North America is Oropsylla montana, a common ground squirrel flea with a wide distribution across western North America. The bacterial communities of $O$. montana and other flea species that transmit $Y$. pestis have previously been characterized (Jones et al., 2008, 2010; Erickson et al., 2009), and it has been suggested that flea-associated bacteria likely interact with $Y$. pestis. However, interactions between flea-associated bacteria and $Y$. pestis have not been previously explored. The purpose of this study was to determine the effects of $Y$. pestis infection on flea-associated bacterial communities.

Rock squirrels, Spermophilus varigatus, were captured, anesthetized and subsequently released from a single colony in Colorado Springs, Colorado on 6 July 2010, and several hundred O. montana were collected. This rock squirrel colony has been a long-term source of $O$. montana for laboratory experiments and neither fleas nor squirrels have ever shown any evidence or symptoms of plague. Upon collection, fleas were immediately placed on 
ice for transport back to the Division of Vector-Borne Disease laboratory in Fort Collins, CO. On 7 July 2010 , the identity of all fleas were confirmed to be $O$. montana based on morphology before further inclusion in the study (Hubbard, 1947; Furman and Catts, 1982).

A subset of fleas $(n=21)$ was stored at $-80{ }^{\circ} \mathrm{C}$ immediately after identification, hereafter referred to as wild fleas. The remaining fleas were kept at $23{ }^{\circ} \mathrm{C}$ and $80 \%$ relative humidity for the remainder of the experiment. On 9 July 2010, approximately two-thirds of the remaining fleas were fed defibrinated rat blood (Bioreclamation, Jericho, NY, USA) spiked with $Y$. pestis strain CO-96-3188 $\left(10^{9}\right.$ cfu $\mathrm{ml}^{-1}$ ) on an artificial feeder. The remaining fleas were fed sterile defibrinated rat blood and used as a negative control. Infected and control fleas were collected between 24 and $96 \mathrm{~h}$ post-feeding, and stored at $-80^{\circ} \mathrm{C}$ until DNA extraction.

DNA was extracted from individual flea samples using the MoBio PowerSoil htp DNA extraction kit (Carlsbad, CA, USA). Bar-coded PCR products were generated as previously described (Jones et al., 2010). Briefly, the bacterial 16S rRNA gene was amplified in triplicate for each sample using $27 \mathrm{f}$ and $338 \mathrm{r}$ primers with 454 adaptors and a unique $12 \mathrm{bp}$ error-correcting barcode under the following conditions: $94{ }^{\circ} \mathrm{C}$ for $5 \mathrm{~min}$; 35 cycles of $94{ }^{\circ} \mathrm{C}$ for $45 \mathrm{~s} ; 50^{\circ} \mathrm{C}$ for $30 \mathrm{~s} ; 72^{\circ} \mathrm{C}$ for $90 \mathrm{~s} ; 72{ }^{\circ} \mathrm{C}$ for $10 \mathrm{~min}$. PCR products from the three reactions were combined, purified using the MoBio UltraClean-htp 96 kit (Carlsbad, CA, USA), and DNA concentrations were estimated using the Invitrogen Quant-IT picogreen dsDNA assay kit (Carlsbad, CA, USA). The EnGenCore Sequencing Facility (Columbia, SC, USA) generated DNA sequences using GS FLX Titanium reagents.

DNA sequence data was processed using QIIME v1.2 (Caporaso et al., 2010). Low-quality sequences were removed using QIIME's default settings. Sequences were combined into phylotypes based on $97 \%$ sequence similarity using the uclust algorithm. The most abundant sequence for each phylotype was selected as the phylotype's representative sequence, and representative sequences were identified using the RDP classifier (http:// rdp.cme.msu.edu) and further classified, if necessary, using BLAST against the GenBank database (http://www.ncbi.nlm.nih.gov). We also generated a phylogenetic tree of representative sequences using FastTree as implemented in QIIME (Price et al., 2009). Y. pestis sequences were removed from the data set. Phylotypes that comprised $<0.5 \%$ of a single flea's bacterial community were removed from that sample, and fleas with $<100$ total DNA sequences were removed from the data set. Fleas that harbored Wolbachia were removed from further analyses because Wolbachia dominated communities when present ( $>65 \%$ average relative abundance), and prevalence varied greatly among treatments $(9.5 \%$ of wild fleas, $35.7 \%$ of control fleas and $45.5 \%$ of infected fleas).
We rarefied the data set such that each sample was represented by 100 DNA sequences. We used two different analyses to generate pairwise distances between all individual bacterial communities: (1) Bray-Curtis transformations of phylotype abundance data (Bray and Curtis, 1957) and (2) Weighted UniFrac distances based on the phylogenetic tree (Lozupone et al., 2007). To determine whether the communities significantly differed from each other, we compared within and among-treatment distances using a $t$-test. We used the entire data set (that is, not rarefied) to test for changes in relative abundances of bacterial groups among treatments using a one-way ANOVA coupled with Tukey's test.

After removing low-quality sequences, Y. pestis sequences and very rare phylotypes, 133024 bacterial $16 \mathrm{~S}$ rRNA gene sequences remained; these sequences separated into 259 phylotypes (FR874046-FR874092; HE660147-HE660360). On average, 1916 sequences were recovered from each wild flea $(n=21), 1774$ sequences were recovered from each control flea $(n=28)$ and 784 sequences were recovered from each infected flea $(n=55)$. The vast majority of detected bacteria belonged to four bacterial phyla: Actinobacteria, Bacteroidetes, Firmicutes and Proteobacteria. These four phyla accounted for $>96 \%$ of detected bacteria in all three treatments. After removing Wolbachia-infected fleas, 67 fleas remained for further analyses (wild, $n=19$; control, $n=18$; infected, $n=30$ ).

Bray-Curtis and UniFrac distances led to identical inferences. Within treatment, distances did not significantly differ between wild fleas (Bray-Curtis: 0.4908; UniFrac: 0.1517, on average) and control fleas (Bray-Curtis: 0.4694; UniFrac: 0.1447, on average), but bacterial communities within infected fleas (Bray-Curtis: 0.2596; UniFrac: 0.0707, on average) were significantly more similar relative to wild and control fleas (Figure 1). This suggests that infection with $Y$. pestis alters the flea-associated bacterial community in a directed manner such that a specific suite of bacteria are lost from the community, whereas another subset of community members is able to persist despite infection. The control versus infected dissimilarity values were also significantly lower than within wild and within control values, but significantly higher than within infected values (Figure 1). This suggests that although laboratory conditions do reduce the bacterial community diversity and variability, the effects are less severe in comparison to $Y$. pestis infection.

Analysis of Variance results indicate that specific bacterial groups respond differently to $Y$. pestis infection and laboratory conditions. At the phylum level, the abundance of Firmicutes was severely reduced in infected fleas, the abundance of Proteobacteria increased in infected fleas and Bacteroidetes were almost completely eliminated in both the infected and control treatments (Table 1). The abundances of some lineages decreased in both 

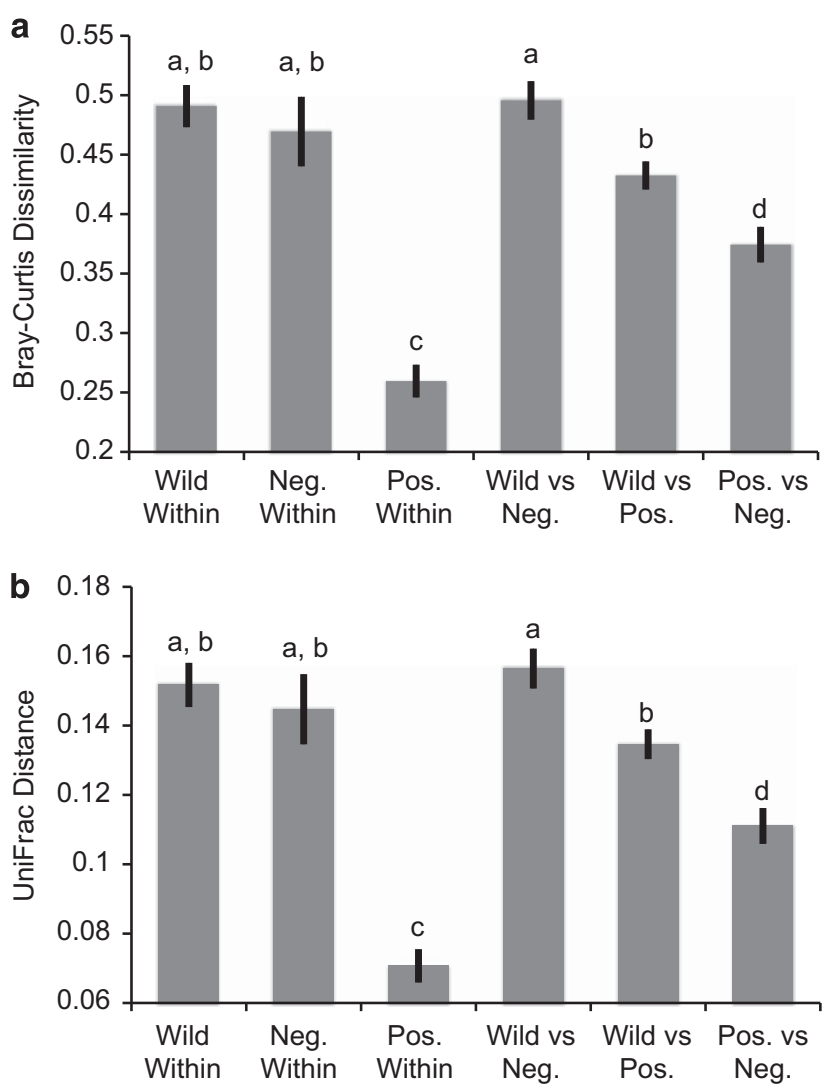

Figure 1 Bray-Curtis dissimilarities (a) and UniFrac distances (b) within and among treatments. Significance was assessed using a $t$-test and $P$-values were adjusted for multiple tests $(P<0.0033=$ significant $)$

control and infected fleas, including Micrococcinae, Streptomycinae, Staphylococcus and Clostridiales; however, in every case, the abundances in the infected fleas decreased more than in the control fleas (Table 1). This indicates that even though some lineages respond negatively to laboratory conditions, $Y$. pestis infection accelerates the changes in composition. Enterococcus abundance only decreased significantly in infected fleas and Bartonella abundance only increased significantly in infected fleas (Table 1).

Lineages that decreased in both $Y$. pestis-positive and $Y$. pestis-negative fleas may have responded to changes in environmental conditions because of the blood meal. In mosquitoes, the gut has been shown to be a reducing environment after feeding on blood (Peterson et al., 2007), and bacterial diversity is initially reduced in mosquito guts after blood feeding (Wang et al., 2011). However, in blood-fed mosquitoes, diversity recovers within 4 days after feeding, and we did not find that in our control or infected fleas. Alternatively, the innate immune response of rat blood may differ from rock-squirrel blood, which may alter the bacterial communities after feeding.

Interestingly, the relative abundance of Grampositive groups (Firmicutes and Actinobacteria) decreased in infected fleas whereas the relative abundance of Gram-negative (Proteobacteria) increased. The reasons for this observation remain unclear and need to be further investigated. The action of pesticin (Pst), a bacteriocin expressed by Y. pestis that disrupts bacterial cell wall integrity by destabilizing peptidoglycan (Vollmer et al., 1997), is unlikely to explain the decreased abundance of Gram-positive lineages observed in our study, because these bacteria are unaffected by this bacteriocin. Many Gram-negative bacteria also appear unaffected by pesticin, although it could potentially impact populations of $Y$. pestis or a few closely related bacteria that are susceptible to its action (Brubaker, 1991). Regardless, the effect, if any, of pesticin on bacterial communities in fleas will need to be tested directly by comparing the response of bacterial lineages in fleas to wild-type and pesticin-free $Y$. pestis strains. Other possible explanations for reduced bacterial diversity after $Y$. pestis infection include: (1) an unidentified bacteriocin that targets non-Yersinia or Grampositive bacterial cells, (2) the use of quorum sensing by $Y$. pestis to 'turn off' co-occurring bacteria or (3) infection alters the bacterial community indirectly by triggering a physiological or immune response of the flea to select for a subset of present bacteria.

Our findings of the flea-associated bacterial community response to $Y$. pestis infection mirror previous studies of the responses of vertebrate gut bacteria and insect-associated bacteria to experimental pathogen infection. In mice, experimental infection of Helicobacter hepaticus altered the bacterial community structure of the cecum and reduced its overall bacterial diversity (Kuehl et al., 2005). In chickens, infection of Campylobacter jejuni resulted in the loss of specific bacterial lineages and more similar bacterial communities among infected chickens relative to control chickens (Johansen et al., 2006). Similar effects have also been seen in other insects; for example, infection of antibiotic-secreting bacteria from the Bacillus cereus group reduced the diversity of gut flora in moth larvae (Raymond et al., 2008).

Overall, we find that flea-associated bacterial communities among infected fleas have much less variation in community composition than among wild or control fleas. Lineage-specific responses to $Y$. pestis infection and laboratory conditions are responsible for differences in infected and control flea communities relative to wild flea-associated bacteria. Two lineages, Bartonella and Wolbachia, appeared to be unaffected or positively affected by $Y$. pestis infection, and these are the most likely lineages to limit infection within fleas under natural conditions. In the future, laboratory conditions may need to be optimized to retain the natural diversity of flea-associated bacteria, if interactions among bacteria and $Y$. pestis are of interest. 
Table 1 Average relative abundances of bacterial groups and Analysis of Variance significance in wild, control and Yersinia pestisinfected Oropsylla montana

\begin{tabular}{|c|c|c|c|c|c|c|}
\hline & \multicolumn{3}{|c|}{ Average relative abundance } & \multicolumn{3}{|c|}{ ANOVA significance } \\
\hline & Wild $(\mathrm{N}=19)$ & Control $(\mathrm{N}=18)$ & Infected $(\mathrm{N}=30)$ & Wild vs control & Wild vs infected & Control vs Infected \\
\hline \multicolumn{7}{|l|}{ Actinobacteria } \\
\hline Corynebacterineae & $0.5 \%$ & $2.0 \%$ & $0.3 \%$ & NS & NS & NS \\
\hline Micrococcinae & $4.1 \%$ & $1.1 \%$ & $0.5 \%$ & $<0.01$ & $<0.01$ & NS \\
\hline Propionibacterineae & $3.5 \%$ & $2.8 \%$ & $3.0 \%$ & NS & NS & NS \\
\hline Streptomycineae & $3.3 \%$ & $0.6 \%$ & $0.2 \%$ & $<0.01$ & $<0.01$ & NS \\
\hline Other & $0.2 \%$ & $<0.1 \%$ & $0.8 \%$ & NS & NS & NS \\
\hline Total Actinobacteria & $11.6 \%$ & $6.5 \%$ & $4.8 \%$ & NS & NS & NS \\
\hline \multicolumn{7}{|l|}{ Bacteroidetes } \\
\hline Sphingobacteriales & $1.7 \%$ & ND & ND & - & - & - \\
\hline Other & $3.5 \%$ & $0.2 \%$ & $0.2 \%$ & $<0.01$ & $<0.01$ & NS \\
\hline Total Bacteroidetes & $5.3 \%$ & $0.2 \%$ & $0.2 \%$ & $<0.01$ & $<0.01$ & NS \\
\hline \multicolumn{7}{|l|}{ Firmicutes } \\
\hline \multicolumn{7}{|l|}{ Bacilli } \\
\hline Enterococcus & $3.2 \%$ & $12.1 \%$ & $0.4 \%$ & NS & NS & $<0.05$ \\
\hline Staphylococcus & $8.3 \%$ & $3.2 \%$ & $0.6 \%$ & $<0.05$ & $<0.01$ & NS \\
\hline Other & $0.5 \%$ & $0.3 \%$ & $0.2 \%$ & NS & NS & NS \\
\hline \multicolumn{7}{|l|}{ Clostridia } \\
\hline Clostridiales & $0.6 \%$ & $0.2 \%$ & $<0.1 \%$ & $<0.01$ & $<0.01$ & $<0.01$ \\
\hline Total Firmicutes & $12.6 \%$ & $15.7 \%$ & $1.2 \%$ & NS & $\mathrm{NS}^{\mathrm{a}}$ & $<0.05^{\mathrm{a}}$ \\
\hline \multicolumn{7}{|l|}{ Proteobacteria } \\
\hline \multicolumn{7}{|l|}{ Alphaproteobacteria } \\
\hline Bartonella & $62.4 \%$ & $71.9 \%$ & $87.5 \%$ & NS & $<0.05^{\mathrm{a}}$ & $\mathrm{NS}^{\mathrm{a}}$ \\
\hline Other & $0.6 \%$ & $0.8 \%$ & $0.3 \%$ & NS & NS & NS \\
\hline \multicolumn{7}{|l|}{ Betaproteobacteria } \\
\hline Burkholderiales & $3.3 \%$ & $1.6 \%$ & $1.6 \%$ & NS & NS & NS \\
\hline \multicolumn{7}{|l|}{ Deltaproteobacteria } \\
\hline \multirow{2}{*}{\multicolumn{7}{|c|}{ Gammaproteobacteria }} \\
\hline & & & & & & \\
\hline Pseudomonadales & $1.3 \%$ & $1.0 \%$ & $0.4 \%$ & NS & NS & NS \\
\hline Other & $<0.1 \%$ & $0.2 \%$ & $0.2 \%$ & NS & NS & NS \\
\hline Total Proteobacteria & $67.7 \%$ & $75.4 \%$ & $89.9 \%$ & NS & $<0.05^{\mathrm{a}}$ & $\mathrm{NS}^{\mathrm{a}}$ \\
\hline \multicolumn{7}{|l|}{ Tenericutes } \\
\hline Spiroplasma & $<0.1 \%$ & ND & $2.4 \%$ & - & NS & - \\
\hline Other Bacteria & $2.7 \%$ & $2.2 \%$ & $1.5 \%$ & NS & NS & NS \\
\hline
\end{tabular}

Abbreviations: ND, not detected; NS, not significant.

${ }^{a}$ Wild + control vs infected also significantly different.

\section{References}

Bray JR, Curtis JT. (1957). An ordination of the upland forest communities of southern Wisconsin. Ecol Monogr 27: 325-349.

Brubaker RR. (1991). Factors promoting acute and chronic diseases caused by Yersiniae. Clin Microbiol Rev 4: 309-324.

Caporaso JG, Kuczynski J, Stombaugh J, Bittinger K, Bushman FD, Costello EK et al. (2010). QIIME allows analysis of high-throughput community sequencing data. Nat Methods 7: 335-336.

Erickson DL, Anderson NE, Cromar LM, Jolley A. (2009). Bacterial communities associated with flea vectors of plague. J Med Entomol 46: 1532-1536.

Eskey CR, Haas VH. (1940). Plague in rodents and fleas in the western part of the United States. Public Health Bulletin.
Furman D, Catts E. (1982). Manual of Medical Entomology, 4th edn, Cambridge University Press: London.

Gage KL, Kosoy MY. (2005). Natural history of plague: perspectives from more than a century of research. Annu Rev Entomol 50: 505-528.

Hubbard C. (1947). Fleas of Western North America. Iowa State College Press: Ames, Iowa.

Johansen CH, Bjerrum L, Finster K, Pedersen K. (2006). Effects of a Campylobacter jejuni infection on the development of the intestinal microflora of broiler chickens. Poult Sci 85: 579-587.

Jones R, Bernhardt S, Martin A, Gage K. (2012). Interactions among symbionts of Oropsylla spp. (Siphonoptera: Ceratophyllidae). J Med Entomol 49: 492-496.

Jones RT, McCormick KF, Martin AP. (2008). Bacterial communities of Bartonella-positive fleas: diversity and community assembly patterns. Appl Environ Microbiol 74: 1667-1670. 
Jones RT, Knight R, Martin AP. (2010). Bacterial communities of disease vectors sampled across time, space, and species. ISME J 4: 223-231.

Korzun VM, Nikitin AI. (1997). Asymmetry of the chaetae in fleas (Citellophilus tesquorum) as a possible marker of their capacity for blocking. Med Parazitol (Mosk) 66: 34-36.

Kuehl CJ, Wood HD, Marsh TL, Schmidt TM, Young VB. (2005). Colonization of the cecal mucosa by Helicobacter hepaticus impacts the diversity of the indigenous microbiota. Infect Immun 73: 6952-6961.

Lozupone CA, Hamady M, Kelley ST, Knight R. (2007). Quantitative and qualitative beta diversity measures lead to different insights into factors that structure microbial communities. Appl Environ Microbiol 73: 1576-1585.

Peterson TML, Gow AJ, Luckhart S. (2007). Nitric oxide metabolites induced in Anopheles stephensi control malaria parasite infection. Free Radic Biol Med 42: 132-142.

Price MN, Dehal PS, Arkin AP. (2009). FastTree: Computing large minimum evolution trees with profiles instead of a distance matrix. Mol Biol Evol 26: 1641-1650.

Raymond B, Lijek RS, Griffiths RI, Bonsall MB. (2008). Ecological consequences of ingestion of Bacillus cereus on Bacillus thuringiensis infections and on the gut flora of a lepidopteran host. J Invertebr Pathol 99: 103-111.

Vollmer W, Pilsl H, Hantke K, Holtje JV, Braun V. (1997). Pesticin displays muramidase activity. J Bacteriol 179: 1580-1583.

Wang Y, Gilbreath TM III, Kukutla P, Yan G, Xu J. (2011). Dynamic gut microbiome across life history of the malaria mosquito anopheles gambiae in Kenya. PLoS One 6: e24767. 